\title{
Early Immunoparalysis Was Associated with Poor Prognosis in Elderly Patients with Sepsis: Secondary Analysis of the ETASS Study
}

This article was published in the following Dove Press journal: Infection and Drug Resistance

Fei Pei, (DD ${ }^{1,2, *}$ Guan-Rong Zhang, ${ }^{3, *}$ Li-Xin Zhou, ${ }^{4}$ Ji-Yun Liu, ${ }^{5}$ Gang Ma, ${ }^{6}$ Qiu-Ye Kou, ${ }^{7}$ Zhi-Jie He, ${ }^{8}$ MinYing Chen,' Yao Nie,' Jian-Feng Wu, ${ }^{1,2}$ Xiang-Dong Guan (iD'

On behalf of the China Critical Care Immunotherapy Research Group

'Department of Critical Care Medicine, The First Affiliated Hospital, Sun Yat-sen University, Guangzhou 510080, People's Republic of China; ${ }^{2}$ Clinical Trial Unit, The First Affiliated Hospital, Sun Yat-sen University \& The University of Birmingham, Guangzhou 510080, People's Republic of China; ${ }^{3}$ Information and Statistics Center, Guangdong Provincial People's Hospital, Guangdong Academy of Medical Sciences, Guangzhou 510080, People's Republic of China; ${ }^{4}$ Department of Critical Care Medicine, Foshan First Municipal People's Hospital, Foshan 528000, People's Republic of China; ${ }^{5}$ Department of Critical Care Medicine, Guangzhou First Municipal People's Hospital, Guangzhou 510180 , People's Republic of China; ${ }^{6}$ Department of Critical Care Medicine, Sun Yat-sen University Cancer Center, Sun Yat-sen University, Guangzhou 510060, People's Republic of China; ${ }^{7}$ Department of Critical Care Medicine, The Sixth Affiliated Hospital, Sun Yat-sen University, Guangzhou 510655, People's Republic of China; ${ }^{8}$ Department of Critical Care Medicine, Sun Yat-sen Memorial Hospital, Sun Yat-sen University, Guangzhou 510120 People's Republic of China

*These authors contributed equally to this work

Correspondence: Jian-Feng Wu Email wujianf@mail.sysu.edu.cn

Xiang-Dong Guan Email guanxiangdong1962@163.com
Purpose: Although immune dysfunction has been investigated in adult septic patients, early immune status remains unclear. In this study, our primary aim was to assess early immune status in adult patients with sepsis stratified by age and its relevance to hospital mortality.

Patients and Methods: A post hoc analysis of a multicenter, randomized controlled trial was conducted; 273 patients whose immune status was evaluated within 48 hours after onset of sepsis were enrolled. Early immune status was evaluated by the percentage of monocyte human leukocyte antigen-DR (mHLA-DR) in total monocytes within 48 hours after onset of sepsis and it was classified as immunoparalysis (mHLA-DR $\leq 30 \%$ ) or non-immunoparalysis $(>30 \%)$. Three logistic regression models were conducted to explore the associations between early immunoparalysis and hospital mortality. We also developed two sensitivity analyses to find out whether the definition of early immune status ( 24 hours vs 48 hours after onset of sepsis) and immunotherapy affect the primary outcome.

Results: Of the 181 elderly ( $\geq 60 \mathrm{yrs})$ and 92 non-elderly (<60yrs) septic patients, $71(39.2 \%)$ and $25(27.2 \%)$ died in hospital, respectively. The percentage of early immunoparalysis in the elderly was twice of that in the non-elderly patients $(32 \%$ vs $16 \%, \mathrm{p}=0.006)$. For the elderly, hospital mortality was higher in the immunoparalysis ones than the nonimmunoparalysis ones $(53.4 \%$ vs $32.5 \%, \mathrm{p}=0.009)$. But there was no significant difference in hospital mortality between immunoparalysis non-elderly patients and nonimmunoparalysis non-elderly ones $(33.5 \%$ vs $26.0 \%, \mathrm{p}=0.541)$. By means of logistic regression models, we found that early immunoparalysis was independently associated with increased hospital mortality in elderly, but not in non-elderly patients. Sensitivity analysis further confirmed the definition of early immune status and immunotherapy did not affect the outcomes.

Conclusion: The elderly were more susceptible to early immunoparalysis after onset of sepsis. Early immunoparalysis was independently associated with poor prognosis in elderly, but not in non-elderly patients.

Keywords: early immune status, elderly, mHLA-DR, immunosuppression, immunoparalysis, sepsis

\section{Introduction}

Sepsis is defined as life-threatening organ dysfunction caused by a dysregulated host response to infection. ${ }^{1}$ After decades of effort, the mortality rate of sepsis has been decreasing; however, the absolute number of deaths is likely to continue to increase as the incidence of sepsis keeps rising. ${ }^{2,3}$ Although sepsis has been studied for decades, its pathogenesis remains unclear. A post-mortem study of septic patients demonstrated 
that patients who died of sepsis were associated with widespread severe immunosuppression. ${ }^{4}$ Subsequently, numerous studies have demonstrated that severe immunosuppression is the main cause of high mortality in septic patients. 5,6

Monocyte human leukocyte antigen-DR (mHLA-DR) is a key biomarker to assess immune status. Our previous study and other studies have revealed that both mHLA-DR and dynamic change of mHLA-DR were associated with poor prognosis in patients with sepsis. ${ }^{7-9}$ The percentage of mHLA-DR lower than or equal to $30 \%$ was widely accepted as immunoparalysis or severe immunosuppression. ${ }^{9-11}$ Furthermore, mHLA-DR has been applied to select severe immunosuppressed septic patients in the study of immunostimulant. $^{12}$ Therefore, the use of mHLA-DR as a biomarker to assess immune status in septic patients is supported by previous research.

It is well known that the immune function declines with age, so the elderly may be more susceptible to early immunoparalysis than younger patients. ${ }^{13,14}$ Elderly patients are more vulnerable to sepsis during hospitalization, and the mortality of elderly septic patients was higher than that of younger patients. ${ }^{15-17}$ Several studies found immunoparalysis in elderly patient increased the risks of death and secondary infection in the course of sepsis. ${ }^{18,19}$ Although immunoparalysis is associated with poor outcome in elderly septic patients, the timing of immunoparalysis remains unclear. Recently, Muszynski et al revealed that critically ill children with sepsis had immunoparalysis/severe immunosuppression from early stage (within 48 hours after onset of sepsis). ${ }^{20}$ However, early immune status of elderly patients with sepsis has not been demonstrated yet.

In our current study, we aimed to assess early immune status in adult patients with sepsis stratified by age and determine their relevance to hospital mortality.

\section{Patients and Methods Study Design}

The ETASS (Efficacy of Thymosin Alpha 1 for Severe Sepsis, ETASS) study was a multi-centre, randomized controlled study comparing the effect of thymosin alpha 1 (T $\alpha 1)$ vs placebo in patients with severe sepsis. ${ }^{21}$ A full description of the methods of the ETASS study, including the full study protocol, case report form, sample size, quality control, and main results can be found in the original paper. ${ }^{21}$ In the ETASS study, severe sepsis was defined as the presence of a proven or suspected infection in at least one site, two or more signs of a systemic inflammatory reaction, and at least one acute sepsis-related organ dysfunction. Therefore, the term 'severe sepsis' in our previous study is approximately equal to the definition of sepsis in Sepsis 3.0. ${ }^{1}$ Unless otherwise specified, 'sepsis' was used to replace "severe sepsis" in this study. Immunotherapy in the study was defined as patients have received at least one dose of thymosin alpha 1 in the ETASS study.

In the present study, the primary object was to assess early immune status in adult septic patients and its relevance to hospital mortality. All adult septic patients were divided into elderly and non-elderly group. According to China Country Assessment Report on Ageing and Health from the World Health Organization in 2015, elderly was defined as aged 60 years or over. ${ }^{22}$ According to previous study, early immune status was defined as the immune status within 48 hours after onset of sepsis. ${ }^{20}$ Immune status was measured by the expression of mHLA-DR because of its proven value in septic patients. ${ }^{23}$ The detail method of mHLA-DR measurement had been described in Supplementary File 1 . Therefore, only those patients with mHLA-DR measured within 48 hours after onset of sepsis were enrolled in this study (273/361). To assess different immune status, we divided early immune status into two categories: immunoparalysis $(\leq 30 \%)$ and nonimmunoparalysis (mHLA-DR $>30 \%$ ). ${ }^{10,24}$ Changes in immune status were expressed by the value change in mHLA-DR on day 3 compared with the first measurement. According to our previous study, a change of mHLA-DR value of $4.8 \%$ on day 3 compared to initial measurement allowed discrimination between survivors and nonsurvivors. ${ }^{8}$ Thus, the value change over $4.8 \%$ was defined as immune status improvement, and equal or less than $4.8 \%$ was defined as immune status non-improvement.

In addition, the Acute Physiology and Chronic Health Evaluation II (APACHE II) score with or without the age component and the Sequential Organ Failure Assessment (SOFA) score were recorded during the first 24 hours after onset of sepsis. When using dichotomous variable for logistic analysis, high SOFA score was defined as the SOFA score more than 8 according to the previous study. ${ }^{25}$ Other clinical or laboratory parameters were also recorded at the same time. For prognosis, we assessed hospital mortality, 28-day mortality, ICU mortality, length of ICU stay and mechanical ventilation (MV) support days.

Because we did a secondary selection of patients from the ETASS study, considering of the possible selection bias, the baseline clinical characteristics between included (273/361) and excluded (88/361) patients were compared. 


\section{Statistic Methods}

Continuous variables with normal distribution were summarized as mean (standard deviation, SD) and compared by $t$-test; while non-normal distributed variables were described as median (interquartile range, IQR) and compared by the Wilcoxon rank sum test. Categorical data were presented as frequencies and percentages, and compared with Chisquared tests. Logistic regression analysis was used to evaluate the association between early immunoparalysis and hospital mortality, stratified by age. In model 1 , the crude odd ratios (ORs) and 95\% confidence intervals (CIs) were calculated by entering only the variable for early immunoparalysis. In model 2, data were adjusted for sex, age, preexisting condition, immunotherapy, and SOFA score. Then, we further adjusted for the dichotomous variable of immune status improvement in model 3 . We also developed two sensitivity analyses to find out whether the definition of early immune status (24 hours vs 48 hours after onset of sepsis) and immunotherapy affect the primary outcome. A p-value $<0.05$ (two tailed) was considered statistically significant. All analyses were conducted using IBM SPSS software version 24.0 (IBM Corp., Armonk, NY, USA).

\section{Results}

\section{Baseline Clinical Characteristics of Adult Patients with Sepsis}

Of the 361 patients, 273 patients were enrolled in this study, including 181 elderly and 92 non-elderly (Figure 1). There were no significant differences in most clinical variables between the included and excluded patients except for the prevalence of immunotherapy ( $54 / 88$ vs $127 / 273, p=0.016)$. The excluded patients were more likely to receive immunotherapy than those included (Supplementary Table 1). Baseline clinical characteristic data of included patients are shown in Table 1. The mean APACHE II scores without the age component were 18.3 (7.4) in the elderly and 17.5 (6.9) in the non-elderly. The mean SOFA scores were 8.0 (3.9) in the elderly and 7.5 (3.6) in the non-elderly. There was no difference in the severity of sepsis between the elderly and non-elderly groups, but the elderly group had a higher percentage of pre-existing conditions than non-elderly $(85.1 \%$ vs $70.7 \%, \mathrm{p}=0.006)$.

\section{Outcomes of Adult Patients with Sepsis}

In our study, 71 (39.2\%) elderly and 25 (27.2\%) non-elderly died in hospital (Table 2). The elderly patients received prolonged days of mechanical ventilation support than the non-elderly patients ( 6.2 vs $4.8 ; \mathrm{p}=0.009)$, but there was no difference in the length of ICU stay (Days: 10.3 vs 9.0; $\mathrm{p}=0.103$ ) between elderly and non-elderly patients (Table 2 ).

\section{Immune Status in Adult Patients with Sepsis}

The percentage of mHLA-DR expression in elderly nonsurvivors was lower than that of elderly survivors on day 0 (Median: 37.1, interquartile range [IQR]: 16.5 71.4 vs 51.5, IQR: $31.3 \sim 77.1)$ and day 3 (38.3, IQR: 19.6 70.4

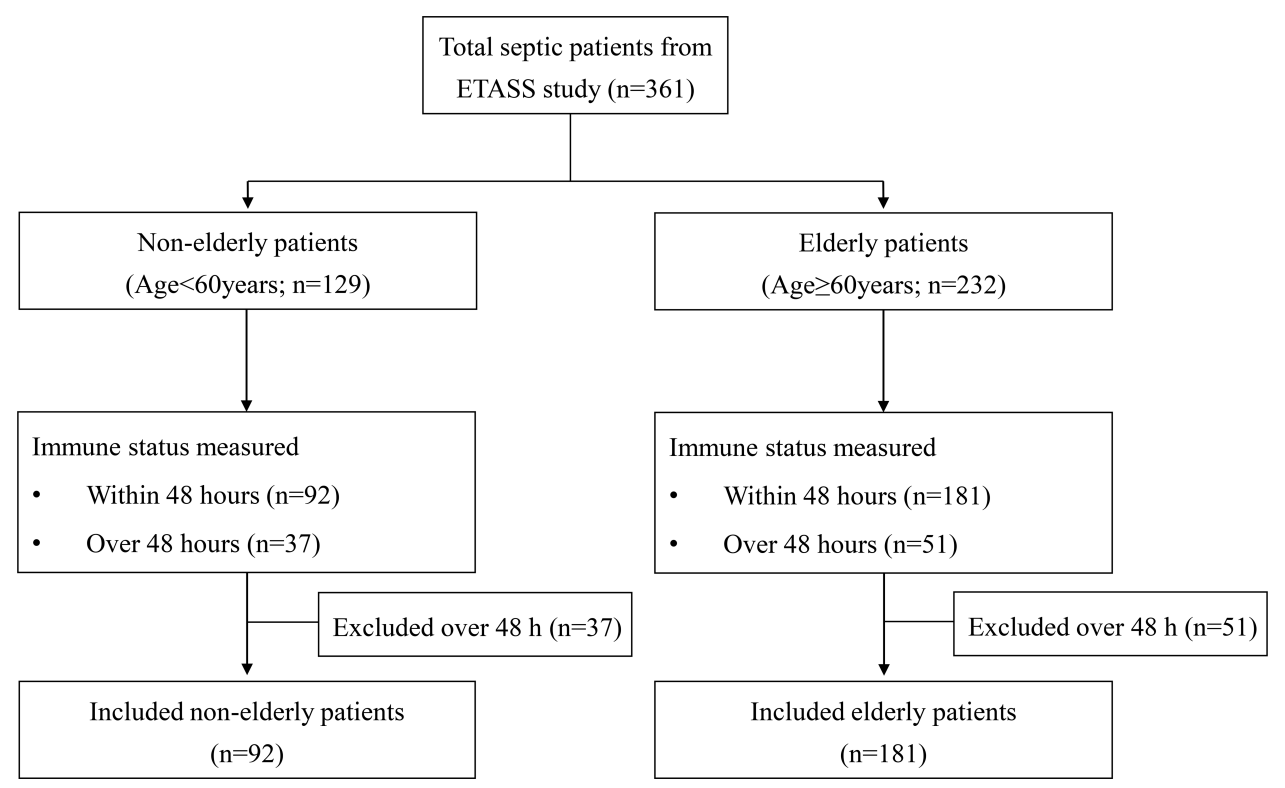

Figure I Flow chart. In this study, I8I elderly and 92 non-elderly septic patients whose mHLA-DR was obtained within 48 hours after onset of sepsis were enrolled. 
Table I Baseline Clinical Characteristics of Adult Patients with Sepsis

\begin{tabular}{|c|c|c|c|}
\hline \multirow[t]{2}{*}{ Characteristics } & \multicolumn{2}{|l|}{$\mathbf{N}(\%)$} & \multirow[t]{2}{*}{$\mathrm{p}$ value } \\
\hline & $\begin{array}{l}\text { Non-Elderly } \\
(<60 \mathrm{yr}, \mathrm{n}=92)\end{array}$ & $\begin{array}{l}\text { Elderly } \\
(\geq 60 \mathrm{yr}, \\
\mathrm{n}=181)\end{array}$ & \\
\hline Age & $54(48,57)$ & $75(68,80)$ & $<0.001$ \\
\hline Sex (male) & $67(72.8)$ & $138(76.2)$ & 0.538 \\
\hline Immunotherapy & $44(48)$ & $83(46)$ & 0.798 \\
\hline Pre-existing conditions & $65(70.7)$ & $154(85.1)$ & 0.006 \\
\hline Congestive cardiomyopathy & $\mathrm{I}(\mathrm{I} .1)$ & $8(4.4)$ & 0.145 \\
\hline Hypertension & $25(27.2)$ & $97(53.6)$ & $<0.001$ \\
\hline Coronary heart disease & $4(4.3)$ & $27(14.9)$ & 0.009 \\
\hline Liver disease & $9(9.8)$ & $3(1.7)$ & 0.002 \\
\hline COPD & $5(5.4)$ & $38(21.0)$ & 0.001 \\
\hline Diabetes & $12(13.0)$ & $41(22.7)$ & 0.058 \\
\hline Recent trauma & $5(5.4)$ & $4(2.2)$ & 0.158 \\
\hline Cancer & $36(39.1)$ & $57(31.5)$ & 0.208 \\
\hline Recent surgical history & & & 0.064 \\
\hline No history of surgery & $40(43.5)$ & $103(56.9)$ & \\
\hline Elective surgery & $24(26.1)$ & $43(23.8)$ & \\
\hline Emergency surgery & $28(30.4)$ & $34(18.8)$ & \\
\hline \multicolumn{4}{|l|}{$\begin{array}{l}\text { Other indicators of disease } \\
\text { severity }\end{array}$} \\
\hline Mechanical ventilation & $64(69.6)$ & $150(82.9)$ & 0.012 \\
\hline Shock & $36(39.1)$ & $72(39.8)$ & 0.917 \\
\hline Use of vasopressor & $37(40.2)$ & $75(41.4)$ & 0.847 \\
\hline RRT & II (12.0) & $27(14.9)$ & 0.312 \\
\hline Low dose corticoid & $12(13.0)$ & $16(8.8)$ & 0.279 \\
\hline Blood transfusion & $36(39.1)$ & $46(25.4)$ & 0.019 \\
\hline \multicolumn{4}{|l|}{ Acute organ dysfunctions } \\
\hline Pulmonary & $85(92.4)$ & $174(96.1)$ & 0.185 \\
\hline Renal & $24(26.1)$ & $51(28.2)$ & 0.715 \\
\hline Cardiovascular & $57(62.0)$ & $133(73.5)$ & 0.050 \\
\hline Haematologic & $42(45.7)$ & $65(35.9)$ & 0.119 \\
\hline Hepatic & $17(18.5)$ & $32(17.7)$ & 0.871 \\
\hline $\begin{array}{l}\text { Number of acute organ } \\
\text { dysfunctions }\end{array}$ & & & 0.531 \\
\hline 1 & $17(18.5)$ & $23(12.7)$ & \\
\hline 2 & $35(38.0)$ & $79(43.6)$ & \\
\hline 3 & $27(29.3)$ & $49(27.1)$ & \\
\hline 4 & $8(8.7)$ & $23(12.7)$ & \\
\hline 5 & $5(5.4)$ & $7(3.9)$ & \\
\hline \multicolumn{4}{|l|}{ Site of infection } \\
\hline Lung & $58(63.0)$ & $14 \mid(77.9)$ & 0.009 \\
\hline Abdomen & $35(38.0)$ & $44(24.3)$ & 0.018 \\
\hline Positive blood culture & $4(4.3)$ & $12(6.6)$ & 0.448 \\
\hline Urinary tract & 0 & $5(2.8)$ & 0.108 \\
\hline Other & $11(12.0)$ & $12(6.6)$ & 0.134 \\
\hline Result of pathogens & & & 0.714 \\
\hline Gram negative & $24(26.1)$ & $37(20.4)$ & \\
\hline Gram positive & $7(7.6)$ & $14(7.7)$ & \\
\hline
\end{tabular}

(Continued)
Table I (Continued).

\begin{tabular}{|c|c|c|c|}
\hline \multirow[t]{2}{*}{ Characteristics } & \multicolumn{2}{|l|}{$\mathbf{N}(\%)$} & \multirow[t]{2}{*}{$\mathrm{p}$ value } \\
\hline & $\begin{array}{l}\text { Non-Elderly } \\
(<60 \mathrm{yr}, \mathrm{n}=92)\end{array}$ & $\begin{array}{l}\text { Elderly } \\
(\geq 60 \mathrm{yr}, \\
n=181)\end{array}$ & \\
\hline Fungus & $7(7.6)$ & $22(12.2)$ & \\
\hline Mixed & 31 (33.7) & $64(35.4)$ & \\
\hline No & $23(25.0)$ & $44(24.3)$ & \\
\hline APACHE II score & $19.6 \pm 6.8$ & $23.5 \pm 7.5$ & $<0.001$ \\
\hline APACHE II score (without age) & $17.5 \pm 6.9$ & $18.3 \pm 7.4$ & 0.378 \\
\hline SOFA score & $7.5 \pm 3.6$ & $8.0 \pm 3.9$ & 0.327 \\
\hline $\mathrm{C}$ reactive protein $(\mathrm{mg} / \mathrm{L})$ & $\begin{array}{l}\text { 127.0 (83.4, } \\
198.0)\end{array}$ & $\begin{array}{l}133.0 \\
(71.2,195.5)\end{array}$ & 0.701 \\
\hline $\begin{array}{l}\text { White blood cell count } \\
\left(\times 10^{9} / L\right)\end{array}$ & $14.1(8.6,19.2)$ & $\begin{array}{l}14.2 \\
(9.8,18.0)\end{array}$ & 0.979 \\
\hline Neutrophil (\%) & $\begin{array}{l}86.3 \\
(80.0,90.7)\end{array}$ & $\begin{array}{l}85.5 \\
(80.7,90.9)\end{array}$ & 0.939 \\
\hline Monocyte (\%) & $4.5(2.9,7.9)$ & $5.0(3.0,7.2)$ & 0.743 \\
\hline Lymphocyte count $\left(\times 10^{9} / \mathrm{L}\right)$ & $\begin{array}{l}0.96 \\
(0.62,1.64)\end{array}$ & $\begin{array}{l}0.84 \\
(0.49,1.29)\end{array}$ & 0.091 \\
\hline Platelet count $\left(\times 10^{9} / \mathrm{L}\right)$ & $\begin{array}{l}160.0 \\
(82.2,282.8)\end{array}$ & $\begin{array}{l}162.7 \\
(101.7,235.3)\end{array}$ & 0.816 \\
\hline Lactate $(\mathrm{mmol} / \mathrm{L})$ & $2.2(1.2,3.5)$ & $2.3(1.4,3.8)$ & 0.304 \\
\hline
\end{tabular}

Note: Values are described by number (percentage), mean \pm standard deviation or median (interquartile range).

Abbreviations: COPD, chronic obstructive pulmonary disease; RRT, renal replacement therapy; APACHE II, Acute Physiology and Chronic Health Evaluation II; SOFA, sequential organ failure assessment.

vs 62.6, IQR: 41.1 82.9) (Figure 2). The mHLA-DR of non-elderly non-survivors was similar to that of nonelderly survivors on day 0 (66.5, IQR: $36.5 \sim 83.8$ vs 61.0, IQR: 39.3 83.8). However, the mHLA-DR of nonelderly non-survivors decreased rapidly on day 3 (46.0, IQR: 20.5 76.7), while that of non-elderly survivors increased slightly (73.1, IQR: 49.4 86.7).

The percentage of early immunoparalysis (mHLA-DR $\leq$ $30 \%)$ in the elderly was twice of that in the non-elderly ( $32 \%$ vs $16 \%, \mathrm{p}=0.006$ ) patients (Figure $3 \mathrm{~A}$ ). The hospital mortality of immunoparalysis elderly patients were higher than that of non-immunoparalysis ones ( $53.4 \%$ vs $32.5 \%, \mathrm{p}=0.009$ ), but there was no significant difference in hospital mortality in the non-elderly between the immunoparalysis and nonimmunoparalysis ( $33.5 \%$ vs $26.0 \%, \mathrm{p}=0.541$ ) (Figure $4 \mathrm{~A}$ ). To detect the change of immune status, 239 septic patients (80 non-elderly and 159 elderly) whose mHLA-DR was measured on day 3 were included. In these patients, about half of the elderly $(82 / 159,52 \%)$ and the non-elderly (38/80, $47 \%$ ) patients had immune status improvement on day 3 (Figure 3B). We also found that patients with immune status improvement on day 3 had lower hospital mortality than those without improvement in both the elderly (21/82 vs 
Table 2 Outcomes of Adult Patients with Sepsis

\begin{tabular}{|l|l|l|l|}
\hline \multirow{2}{*}{ Characteristics } & \multicolumn{2}{|l|}{$\mathbf{N}$ (\%) } & \multirow{2}{*}{ p value } \\
\cline { 2 - 3 } & $\begin{array}{l}\text { Non-Elderly } \\
\mathbf{( < 6 0 y r , ~ n = 9 2 )}\end{array}$ & $\begin{array}{l}\text { Elderly }(\mathbf{6 0} \mathbf{y r}, \\
\mathbf{n = 1 8 1})\end{array}$ & \\
\hline $\begin{array}{l}\text { Hospital mortality } \\
\text { ICU mortality }\end{array}$ & $25(27.2)$ & $71(39.2)$ & 0.049 \\
28-day mortality & $15(16.3)$ & $50(27.6)$ & 0.038 \\
$\begin{array}{l}\text { Days of MV } \\
\text { (Median, IQR) }\end{array}$ & $4.8(1.6,7.7)$ & $62(34.3)$ & 0.081 \\
$\begin{array}{l}\text { MV free days } \\
\text { (Median, IQR) }\end{array}$ & $23.3(20.3,26.4)$ & $21.8(13.8,25.4)$ & 0.009 \\
$\begin{array}{l}\text { ICU stay days } \\
\text { (Median, IQR) }\end{array}$ & $9.0(5.5,14.7)$ & $10.3(5.6,20.7)$ & 0.103 \\
ICU-free days & $19.0(13.3,22.6)$ & $17.8(7.3,22.4)$ & 0.112 \\
(Median, IQR) & & & \\
\hline
\end{tabular}

Notes: Values are described by number (percentage) or median (interquartile range). "Free days" were calculated as the number of days that the patient was alive and free of specified intervention (ventilator use and ICU stay) during the 28day study period.

Abbreviations: IQR, interquartile range; MV, mechanical ventilation.

$35 / 77)$ and the non-elderly (4/38 vs 16/42) groups (Figure 4B).

\section{Early Immunoparalysis Was Associated with Increased Mortality in Elderly Patients}

In univariate logistic regression analysis (model 1), early immunoparalysis was associated with increased hospital mortality in elderly (ORs: 2.382; 95\% CIs: 1.257 4.514; $\mathrm{p}=0.008$ ), but not in non-elderly (ORs: 1.425 ; 95\% CI 0.434-4.676; $\mathrm{p}=0.559$ ) patients (Table 3). After variables of age, sex, pre-existing conditions, immunotherapy and SOFA score being adjusted in model 2 , early immunoparalysis was independently associated with increased hospital mortality in elderly (ORs: 2.257 ; 95\% CI 1.130-4.506; $\mathrm{p}=0.021$ ), but not in non-elderly (ORs: 1.074; 95\% CI 0.242-4.763; $\mathrm{p}=0.925$ )

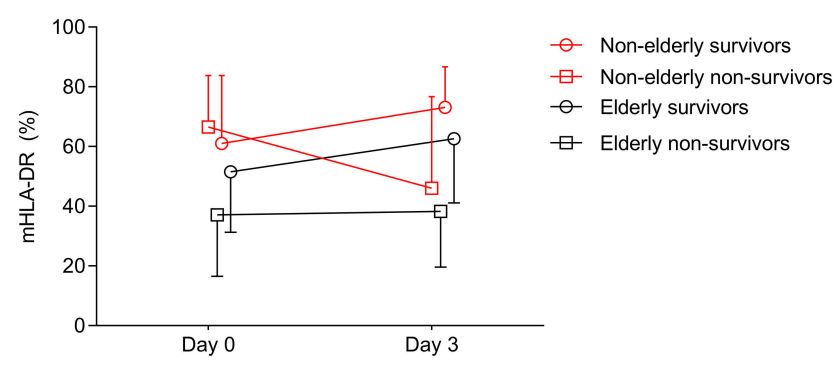

Figure 2 The changes of mHLA-DR in survivors and non-survivors in different age. The mHLA-DR in elderly non-survivors was lower than that of survivors on day 0 and day 3. However, the mHLA-DR of non-elderly non-survivors was similar to that of survivors on day 0 , but mHLA-DR decreased rapidly in non-elderly non-survivors on day 3. patients (Table 3). Then, we conducted another model (model 3) to add the immune status improvement on day 3 into model 2. In model 3, early immunoparalysis was also only associated with increased hospital mortality in elderly (ORs: 2.684; 95\% CIs: 1.224 5.883; $\mathrm{p}=0.014$ ), but not in non-elderly (ORs: 1.604; 95\% CI 0.351-7.331; $\mathrm{p}=0.542$ ) patients (Table 3 ). In addition, we also found that immune status improvement on day 3 was associated with reduced hospital mortality in both elderly (ORs: 0.335 ; 95\% CI 0.159-0.706; $\mathrm{p}=0.004$ ) and non-elderly (ORs: 0.131; 95\% CI 0.029-0.584; $\mathrm{p}=0.008$ ) patients.

The definition of early immune status is a controversial issue, and it was defined as immune status within 48 hours after onset of sepsis in our study. To evaluate whether the definition of early immune status was driving our results, we performed a sensitivity analysis in which early immune status was measured within 24 hours after onset of sepsis. Consistently, early immunoparalysis was independently associated with increased hospital mortality in elderly patients (ORs: 5.507; 95\% CIs: 1.497 20.258; p=0.010), but not in non-elderly (ORs: 1.536 ; 95\% CI 0.236-10.023; $\mathrm{p}=0.654$ ) ones (Supplementary Figure 1A). Then, we also performed another sensitivity analysis to further confirm whether immunotherapy affected our results. We selected patients with sepsis without immunotherapy, and the results were consistent with above that early immunoparalysis was independently associated with increased hospital mortality in elderly patients (ORs: 3.638; 95\% CIs: 1.116 11.852; $\mathrm{p}=0.032$ ), but not in the non-elderly (ORs: 7.002; 95\% CI $0.678-72.325 ; \mathrm{p}=0.102$ ) ones (Supplementary Figure 1B).

\section{Discussion}

The present study is, to the best of our knowledge, the first to evaluate early immune status in elderly septic patients. Our study has a few important findings. First, our data indicated the elderly had greater risk of developing immunoparalysis within 48 hours after onset of sepsis, and the rate was twice of that of the non-elderly. Second, early immunoparalysis in the elderly was associated with poor prognosis, but not in non-elderly patients. Third, immune status improvement was associated with reduced mortality in both the elderly and the non-elderly.

Immunoparalysis/severe immunosuppression was associated with increased mortality and secondary infection, prolonged length of ICU stay and aggravated organ dysfunction in adult and children with sepsis. ${ }^{19,20,26,27}$ However, the initiation of immunoparalysis remains controversial, so it is difficult to determine when to start immune monitoring and 


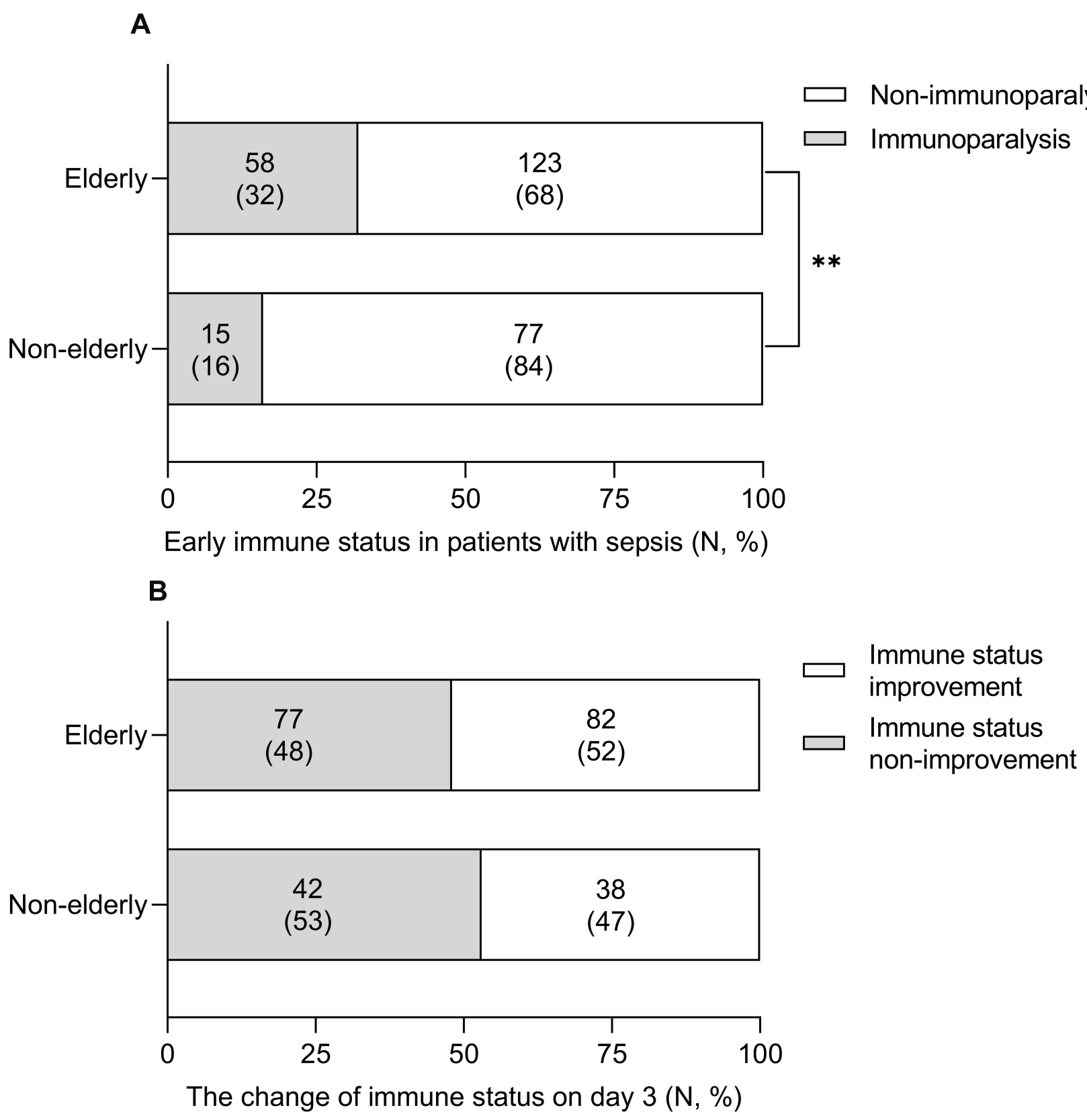

Figure 3 Early immune status and change of immune status in patients with sepsis. (A) The percentage of early immunoparalysis in elderly patients was twice of that of nonelderly patients $(32 \%$ vs $16 \%, p=0.008)$. (B) About half of elderly $(82 / 159,52 \%)$ and non-elderly $(38 / 80,47 \%)$ patients had immune status improvement on day 3 (**p value $<0.01$ ).

administer immunotherapy. A recent study demonstrated that septic paediatric patients were severe immunosuppressed within the first 48 hours after sepsis, and such early immunoparalysis/severe immunosuppression was significantly associated with prolonged organ dysfunction time. ${ }^{20}$ In our study, about one-third of the elderly (58/181) had early immunoparalysis within 48 hours, and more than half of the immunoparalysis patients (31/58) died in hospital. All these results displayed that the elderly were more vulnerable to immune dysfunction early after onset of sepsis. Therefore, the immune status of elderly patients needs to be monitored from the early stage of sepsis.

Consistent with previous studies, we found elderly patients with sepsis have higher mortality than the non-elderly. ${ }^{2,28}$ However, it appears that it is not the age per se but rather the associated factors, such as severity of illness or immune status, contribute to the increased mortality rate. ${ }^{29}$ In our study, the severity of sepsis (SOFA score and APACHE- II score without an age component) was similar in the elderly and nonelderly, but the percentage of early immunoparalysis in the 

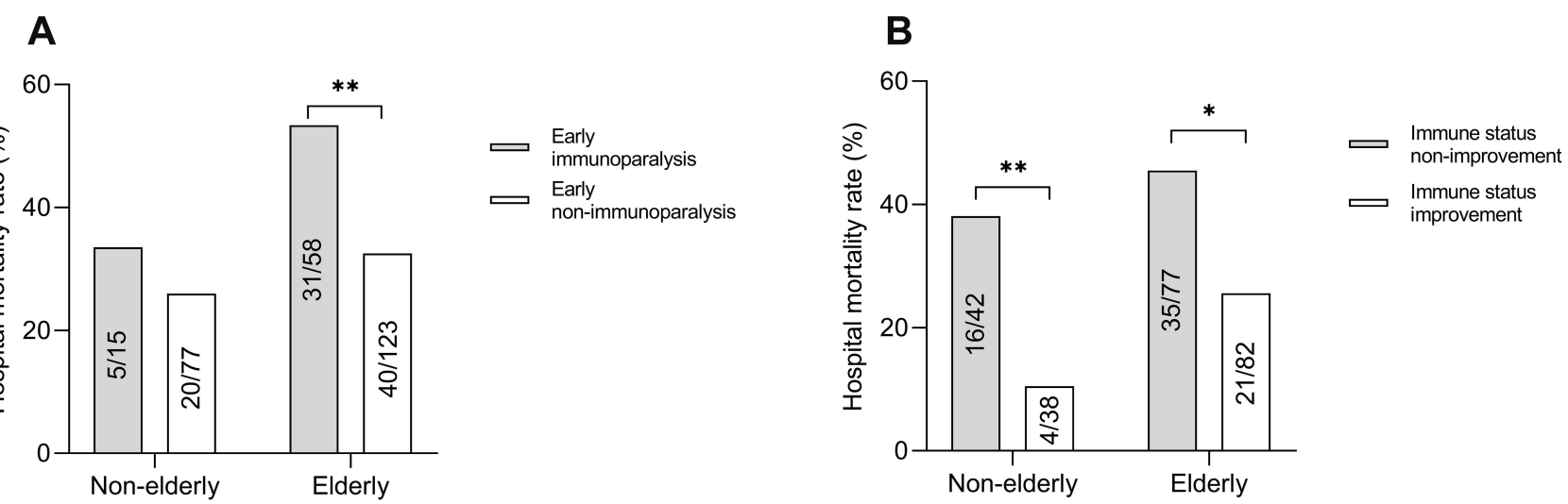

Figure 4 Immune status and hospital mortality. (A) The hospital mortality of immunoparalysis elderly patients were higher than that of non-immunoparalysis ones ( $3 \mathrm{I} / 58$ vs 40/123), but there was no significant difference in hospital mortality in the non-elderly between immunoparalysis and non-immunoparalysis (5/I5 vs 20/77). (B) Septic patients with immune status improvement on day 3 had lower hospital mortality than patients with non-improvement in both the elderly and the non-elderly groups (*P value $<0.05 ; * *$ value $<0.01$ ).

elderly was twice of that in the non-elderly patients. We also found that more than half of the immunoparalysis elderly (31/ $58,53 \%$ ) died in hospital, but only one-third of the non-elderly $(5 / 15,33 \%)$ died during hospitalization. Furthermore, immunoparalysis was associated with increased hospital mortality in the elderly, but not in non-elderly patients. That is to say, immunoparalysis may be responsible for the increased mortality in elderly patients with sepsis.

Numerous studies revealed that mHLA-DR can be used to predict prognosis and to select septic patients who needed immunostimulant. ${ }^{8,9,30}$ Monneret et al previously found that mHLA-DR decreased in septic patients on days 1-2 and days 3-4 after onset of sepsis, but only low mHLA-DR $(\leq 30 \%)$ on days 3-4 was independently associated with increased 28day mortality in patients with sepsis. ${ }^{10}$ However, Perry et al reported a different result that mHLA-DR on days 1-3 cannot help to predict outcome in sepsis. ${ }^{31}$ In Perry's research, the median age of septic patients was about 56 with a range from 20 to 84, while in Monneret's study, the median age was 64 (IQR: 48 75). We speculated that the age differences in septic patients may be the cause of the opposite outcomes in the two studies. In current study, our results indicated that early low mHLA-DR expression was an independent risk factor for poor outcome in elderly, but not in non-elderly septic patients. Our previous study demonstrated that dynamic change of mHLA-DR was a reliable predictor for mortality in septic patients. ${ }^{8}$ Then, we combined early immune status with changes of immune status to evaluate hospital mortality. In this study, early immunoparalysis was associated with increased hospital mortality in the elderly, and immune status improvement on day 3 was associated with reduced hospital mortality in both elderly and nonelderly patients. Therefore, monitoring of early immune status should be carried out in elderly patients, and it may be beneficial to monitor the dynamic changes of immune status in both elderly and non-elderly patients.

Table 3 Early Immunoparalysis Was Associated with Increased Hospital Mortality in Elderly Patients

\begin{tabular}{|c|c|c|c|}
\hline Subgroup & Model I & Model $2^{a}$ & Model $3^{b}$ \\
\hline \multicolumn{4}{|l|}{ Non-elderly } \\
\hline Sample size (n) & 92 & 92 & 80 \\
\hline Early immunoparalysis & $1.425(0.434-4.676)$ & $1.074(0.242-4.763)$ & $\mathrm{I} .604(0.35 \mathrm{I}-7.33 \mathrm{I})$ \\
\hline Immune status improvement & - & - & $0.131(0.029-0.584)$ \\
\hline \multicolumn{4}{|l|}{ Elderly } \\
\hline Sample size (n) & 181 & 181 & 159 \\
\hline Early immunoparalysis & $2.382(|.257-4.5| 4)$ & $2.257(1.130-4.506)$ & $2.684(1.224-5.883)$ \\
\hline Immune status improvement & - & - & $0.335(0.159-0.706)$ \\
\hline
\end{tabular}

Notes: Values are odds ratios (95\% confidence intervals) unless stated otherwise. ${ }^{a}$ Adjusted for sex, age (per 10 years), pre-existing condition, immunotherapy, and SOFA score (high vs low). ${ }^{\mathrm{b}}$ Adjusted for covariates in model 2 and the dichotomous variable of immune status improvement on day 3 (yes vs no). 
Several limitations should be noted in our study. Firstly, our data came from a clinical study of immunotherapy for patients with sepsis. Considering the interference of immunotherapy in our study, we took immunotherapy as a fixed covariate in multivariate logistic regression analysis for mortality and found immunotherapy did not affect the prognosis of elderly patients. Secondly, only 15 non-elderly septic patients with early immunoparalysis were included in our study, so a larger study is needed to further verify the results that early immunoparalysis was not associated with poor prognosis in non-elderly patients.

\section{Conclusions}

In adult patients with sepsis, the elderly were more susceptible to early immunoparalysis after onset of sepsis. Early immunoparalysis was associated with poor prognosis in elderly patients, but not in non-elderly patients. In addition, immune status improvement was associated with reduced mortality in both elderly and non-elderly patients.

\section{Abbreviations}

APACHE II, Acute Physiology and Chronic Health Evaluation II; CIs, confidence intervals; COPD, chronic obstructive pulmonary disease; CRP, C-reactive protein; ETASS, Efficacy of Thymosin Alpha 1 for Severe Sepsis; ICU, intensive care unit; IQR, interquartile range; MAP, mean arterial pressure; mHLA-DR, monocyte human leukocyte antigen-DR; MV, mechanical ventilation; ORs, odds ratios; RRT, renal replacement therapy; SBP, systolic blood pressure; $\mathrm{SD}$, standard deviation; SOFA, sequential organ failure assessment; SSC, surviving sepsis campaign; T $\alpha 1$, thymosin alpha 1.

\section{Data Sharing Statement}

The datasets in this study are available from the corresponding author on reasonable request.

\section{Ethics Approval}

The original study was approved by the Ethics Committee of the First Affiliated Hospital, Sun Yat-sen University, the Ethics Committee of Sun Yat-sen Memorial Hospital, Sun Yat-sen University, the Ethics Committee of Sixth Affiliated Hospital, Sun Yat-sen University, the Ethics Committee of Sun Yat-sen University Cancer Center, Sun Yat-sen University, the Ethics Committee of Foshan First Municipal People's Hospital and the Ethics Committee of Guangzhou First Municipal People's Hospital. Written informed consents were obtained from patients or next of kin for patients unable to consent. The clinical trial registry number is NCT00711620. We make sure to conduct this trial in accordance with the Declaration of Helsinki.

\section{Acknowledgments}

We would like to thank all of the doctors, nurses, technicians, and patients involved at the six participating centres for their dedication to the ETASS study.

\section{Author Contributions}

All authors made substantial contributions to conception and design, acquisition of data, or analysis and interpretation of data; took part in drafting the article or revising it critically for important intellectual content; gave final approval of the version to be published; and agree to be accountable for all aspects of the work.

\section{Funding}

This work was funded by Sun Yat-sen University Clinical Research Program 5010 (2007015, XD Guan), Fundamental Research Funds for the Central Universities (15ykpy14, JF Wu), Natural Science Foundation of Guangdong Province (2016A030313269, JF Wu), and Scientific Research Funds of AESCULAP Academy (K0601288, JF Wu).

\section{Disclosure}

All authors declare that they have no competing interests.

\section{References}

1. Singer M, Deutschman CS, Seymour CW, et al. The third international consensus definitions for sepsis and septic shock (sepsis-3). JAMA. 2016;315(8):801-810. doi:10.1001/jama.2016.0287

2. Gaieski DF, Edwards JM, Kallan MJ, Carr BG. Benchmarking the incidence and mortality of severe sepsis in the United States. Crit Care Med. 2013;41(5):1167-1174. doi:10.1097/CCM.0b013e31827c09f8

3. Suarez De La Rica A, Gilsanz F, Maseda E. Epidemiologic trends of sepsis in western countries. Ann Transl Med. 2016;4(17):325. doi:10.21037/atm.2016.08.59

4. Boomer JS, To K, Chang KC, et al. Immunosuppression in patients who die of sepsis and multiple organ failure. JAMA. 2011;306 (23):2594-2605. doi:10.1001/jama.2011.1829

5. Mira JC, Gentile LF, Mathias BJ, et al. Sepsis pathophysiology, chronic critical illness, and persistent inflammation-immunosuppression and catabolism syndrome. Crit Care Med. 2017;45(2):253-262. doi:10.1097/ CCM.0000000000002074

6. Hotchkiss RS, Monneret G, Payen D. Sepsis-induced immunosuppression: from cellular dysfunctions to immunotherapy. Nat Rev Immunol. 2013;13(12):862-874. doi:10.1038/nri3552

7. Gouel-Cheron A, Allaouchiche B, Floccard B, Rimmele T, Monneret G. Early daily mHLA-DR monitoring predicts forthcoming sepsis in severe trauma patients. Intensive Care Med. 2015;41 (12):2229-2230. doi:10.1007/s00134-015-4045-1 
8. Wu JF, Ma J, Chen J, et al. Changes of monocyte human leukocyte antigen-DR expression as a reliable predictor of mortality in severe sepsis. Crit Care. 2011;15(5):R220. doi:10.1186/cc10457

9. Landelle C, Lepape A, Voirin N, et al. Low monocyte human leukocyte antigen-DR is independently associated with nosocomial infections after septic shock. Intensive Care Med. 2010;36(11):1859-1866. doi:10.1007/s00134-010-1962-x

10. Monneret G, Lepape A, Voirin N, et al. Persisting low monocyte human leukocyte antigen-DR expression predicts mortality in septic shock. Intensive Care Med. 2006;32(8):1175-1183. doi:10.1007/ s00134-006-0204-8

11. Lin HY, Guo XS, Yao YM, et al. Clinical trial to verify the value of the CD14(+) monocyte human leukocyte antigen DR as a marker in evaluating immunosuppression in patients with severe sepsis. Zhongguo Wei Zhong Bing Ji Jiu Yi Xue. 2003;15(3):135-138.

12. Meisel C, Schefold JC, Pschowski R, et al. Granulocyte-macrophage colony-stimulating factor to reverse sepsis-associated immunosuppression: a double-blind, randomized, placebo-controlled multicenter trial. Am J Respir Crit Care Med. 2009;180(7):640-648. doi:10.1164/ rccm.200903-0363OC

13. Caruso C, Buffa S, Candore G, et al. Mechanisms of immunosenescence. Immun Ageing. 2009;6(1):10. doi:10.1186/ 1742-4933-6-10

14. Martin S, Perez A, Aldecoa C. Sepsis and immunosenescence in the elderly patient: a review. Front Med (Lausanne). 2017;4:20. doi:10.3389/fmed.2017.00129

15. Dombrovskiy VY, Martin AA, Sunderram J, Paz HL. Rapid increase in hospitalization and mortality rates for severe sepsis in the United States: a trend analysis from 1993 to 2003. Crit Care Med. 2007;35 (5):1244-1250. doi:10.1097/01.CCM.0000261890.41311.E9

16. Baykara N, Akalin H, Arslantas MK, et al. Epidemiology of sepsis in intensive care units in Turkey: a multicenter, point-prevalence study. Crit Care. 2018;22(1):93. doi:10.1186/s13054-018-2013-1

17. Lee SH, Hsu TC, Lee MG, et al. Nationwide trend of sepsis: a comparison among octogenarians, elderly, and young adults. Crit Care Med. 2018;46 (6):926-934. doi:10.1097/CCM.0000000000003085

18. Inoue S, Suzuki-Utsunomiya K, Okada Y, et al. Reduction of immunocompetent $\mathrm{T}$ cells followed by prolonged lymphopenia in severe sepsis in the elderly. Crit Care Med. 2013;41(3):810-819. doi:10.1097/CCM.0b013e318274645f

19. Suzuki K, Inoue S, Kametani Y, et al. Reduced immunocompetent $\mathrm{B}$ cells and increased secondary infection in elderly patients with severe sepsis. Shock. 2016;46(3):270-278. doi:10.1097/SHK.000 0000000000619
20. Muszynski JA, Nofziger R, Moore-Clingenpeel M, et al. Early immune function and duration of organ dysfunction in critically iii children with sepsis. Am J Respir Crit Care Med. 2018;198 (3):361-369. doi:10.1164/rccm.201710-2006OC

21. Wu J, Zhou L, Liu J, et al. The efficacy of thymosin alpha 1 for severe sepsis (ETASS): a multicenter, single-blind, randomized and controlled trial. Crit Care. 2013;17(1):R8. doi:10.1186/cc11932

22. World Health Organization. China country assessment report on ageing and health. 2015. Available from: http://www.who.int/ageing/pub lications/china-country-assessment/en/. Accessed October 13, 2019 ..

23. Pfortmueller CA, Meisel C, Fux M, Schefold JC. Assessment of immune organ dysfunction in critical illness: utility of innate immune response markers. Intensive Care Med Exp. 2017;5(1):49. doi:10.1186/s40635-017-0163-0

24. Volk HD, Reinke P, Krausch D, et al. Monocyte deactivation-rationale for a new therapeutic strategy in sepsis. Intensive Care Med. 1996;22(Suppl 4):S474-S481. doi:10.1007/BF01743727

25. Ferreira FL, Bota DP, Bross A, Melot C, Vincent JL. Serial evaluation of the SOFA score to predict outcome in critically ill patients. JAMA. 2001;286(14):1754-1758. doi:10.1001/jama.286.14.1754

26. Remy S, Kolev-Descamps K, Gossez M, et al. Occurrence of marked sepsis-induced immunosuppression in pediatric septic shock: a pilot study. Ann Intensive Care. 2018;8(1):36. doi:10.1186/s13613-0180382-x

27. Hotchkiss RS, Monneret G, Payen D. Immunosuppression in sepsis: a novel understanding of the disorder and a new therapeutic approach. Lancet Infect Dis. 2013;13(3):260-268. doi:10.1016/ S1473-3099(13)70001-X

28. Biston P, Aldecoa C, Devriendt J, et al. Outcome of elderly patients with circulatory failure. Intensive Care Med. 2014;40(1):50-56. doi:10.1007/s00134-013-3121-7

29. Flaatten H, de Lange DW, Artigas A, et al. The status of intensive care medicine research and a future agenda for very old patients in the ICU. Intensive Care Med. 2017;43(9):1319-1328. doi:10.1007/ s00134-017-4718-z

30. Lukaszewicz AC, Grienay M, Resche-Rigon M, et al. Monocytic HLA-DR expression in intensive care patients: interest for prognosis and secondary infection prediction. Crit Care Med. 2009;37 (10):2746-2752. doi:10.1097/CCM.0b013e3181ab858a

31. Perry SE, Mostafa SM, Wenstone R, Shenkin A, McLaughlin PJ. Is low monocyte HLA-DR expression helpful to predict outcome in severe sepsis? Intensive Care Med. 2003;29(8):1245-1252. doi:10.1007/s00134-003-1686-2
Infection and Drug Resistance

\section{Publish your work in this journal}

Infection and Drug Resistance is an international, peer-reviewed openaccess journal that focuses on the optimal treatment of infection (bacterial, fungal and viral) and the development and institution of preventive strategies to minimize the development and spread of resistance. The journal is specifically concerned with the epidemiology of antibiotic resistance and the mechanisms of resistance development and diffusion in both hospitals and the community. The manuscript management system is completely online and includes a very quick and fair peerreview system, which is all easy to use. Visit http://www.dovepress.com/ testimonials.php to read real quotes from published authors. 